\title{
Performances techniques et environnementales du four de carbonisation Green Mad Retort à Madagascar
}

\section{Michaël TeMMERMAN ${ }^{1}$ Ravo ANDRIANIRINA ${ }^{2}$ Frank RICHTER ${ }^{1}$}

${ }^{1}$ Eco Consulting Group GmbH \& Co. KG Hersfelder Straße 17

36820 Oberaula

Allemagne

\section{${ }^{2}$ ECO-DIANA}

Villa Lotus bleu Lot 1 -A Bis

SA Scama 201

Antsiranana

Madagascar

\section{Auteur correspondant /} Corresponding author: Michaël TEMMERMAN mike.temmerman@eco-consult.com

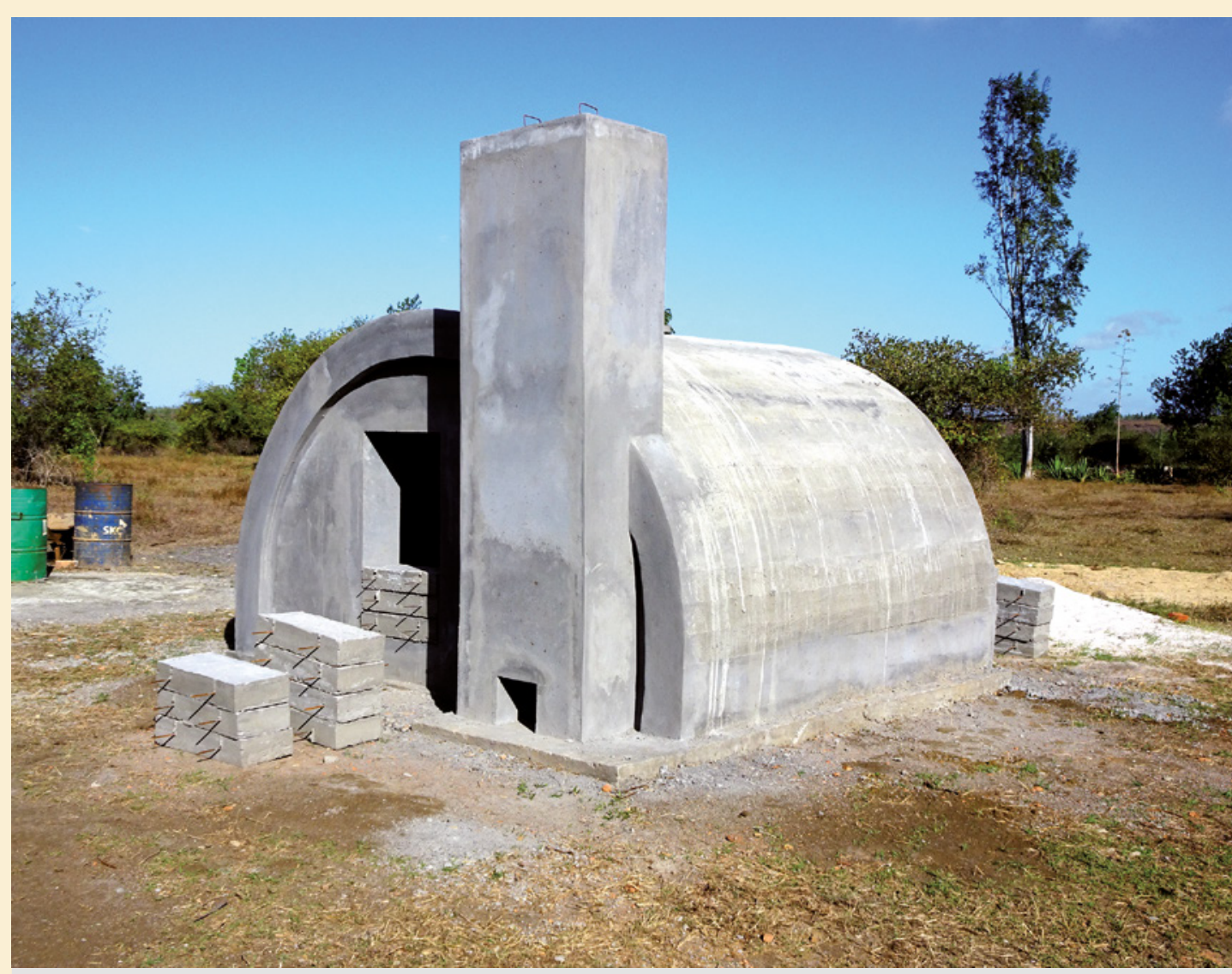

Photo 1.

Four de carbonisation GMDR 3.

Photo M. Temmerman.

Doi : 10.19182/bft2019.340. a31700 - Droit d'auteur (c) 2019, Bois et Forêts des Tropiques @ Cirad - Date de soumission : 29 septembre 2018 ; date d'acceptation : 8 janvier 2019; date de publication : $1^{\text {er }}$ avril 2019.

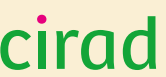

Citer l'article / To cite the article

Temmerman M., Andrianirina R., Richter F., 2019. Performances techniques et environnementales du four de carbonisation Green Mad Retort à Madagascar. Bois et Forêts des Tropiques, 340 : 43-55. Doi : https://doi. $\mathrm{org} / 10.19182 / \mathrm{bft} 2019.340 . \mathrm{a} 31700$ 


\section{RÉSUMÉ}

\section{Performances techniques et environnementales du four de carbonisation Green Mad Retort à Madagascar}

Des essais de carbonisation d'eucalyptus issus de plantations au nord de Madagascar ont été menés sur le terrain dans des fours de carbonisation à chambre de combustion externe (Green Mad Retort ou GMDR). Les analyses d'une cinquantaine d'essais démontrent que des rendements massiques élevés (34\%) et très stables sont obtenus avec ces fours, même pour des charges à l'humidité élevée. L'étude confirme le lien direct entre augmentation du rendement massique et diminution des émissions de gaz à effet de serre liés à la carbonisation $\left(\mathrm{CO}_{2}\right.$ et $\left.\mathrm{CH}_{4}\right)$. Il est également démontré, grâce à des mesures réalisées sur deux fours à des stades de développement différents, qu'il est possible et utile d'éliminer la majeure partie des émissions de méthane à la sortie de la chambre de carbonisation. Dans ce cas, les émissions de méthane sont de l'ordre de 4,3 kg par tonne de charbon de bois produite, ce qui est 5 à 17 fois moins élevé que les émissions publiées pour d'autres techniques de carbonisation, à l'exception des fours cornues industriels.

Mots-clés : carbonisation, effet de serre, émissions de méthane, eucalyptus, rendement de carbonisation, techniques améliorées de carbonisation, Madagascar.

\section{ABSTRACT}

\section{RESUMEN}

\section{Technical and environmental performance of the Green Mad Retort charcoal-making kiln in Madagascar}

Charcoal-making trials with eucalyptus from plantations in northern Madagascar were conducted in the field using Green Mad Retort (GMDR) external combustion chamber kilns. Analyses of some fifty trials show that these kilns produce high (34\%) and very stable mass yields, even from wood with a high moisture content. The study confirms the direct link between higher mass yields and lower greenhouse gas emissions from charcoal-making $\left(\mathrm{CO}_{2}\right.$ and $\mathrm{CH}_{4}$ ). Measurements made in two kilns at different stages in development also show that it is possible to eliminate most methane emissions at the outflow from the combustion chamber. In this case, methane emissions are around $4.3 \mathrm{~kg} / \mathrm{t}$ of charcoal produced, which is 5 to 17 times lower than published emission figures for other carbonisation techniques, with the exception of industrial retort furnaces.

Keywords: carbonisation, greenhouse effect, methane emissions, Eucalyptus, charcoal-making yields, improved charcoal-making techniques, Madagascar.

\section{Rendimiento técnico y medioambiental del horno de carbonización Green Mad Retort en Madagascar}

Se han llevado a cabo sobre el terreno ensayos de carbonización de eucaliptos provenientes de plantaciones del norte de Madagascar en hornos de carbonización con cámara de combustión externa (Green Mad Retort o GMDR). Los análisis de una cincuentena de ensayos demuestran que se han obtenido rendimientos másicos elevados (34 \%) y muy estables con estos hornos, incluso para cargas con humedad elevada. El estudio confirma la relación directa entre el aumento del rendimiento másico y la disminución de emisiones de gas con efecto invernadero provenientes de la carbonización $\left(\mathrm{CO}_{2}\right.$ y $\left.\mathrm{CH}_{4}\right)$. Se ha demostrado también, gracias a las medidas realizadas en dos hornos en estadios de desarrollo diferentes, que es posible y útil eliminar la mayor parte de las emisiones de metano a la salida de la cámara de carbonización. En este caso, las emisiones de metano son del orden de 4,3 kg/t de carbón de leña producido, lo que es de 5 a 17 veces menor que las emisiones publicadas para otras técnicas de carbonización, exceptuando los hornos de retorta industriales.

Palabras clave: carbonización, efecto invernadero, emisiones de metano, eucalipto, rendimiento de carbonización, técnicas mejoradas de carbonización, Madagascar. 


\section{Introduction}

Les techniques de carbonisation sont classiquement regroupées en fours à combustion partielle de la charge, fours cornues et fours à contact de gaz chauds (Schenkel, 1999 ; Girard et Napoli, 2005 ; Riuji Lohri et al., 2016). Les premiers peuvent encore être subdivisés selon que le tirage est direct ou inversé (Sanogo et al., 2006). Les rendements de ces différentes techniques ont fait l'objet de publications synthétisées dans le tableau I. Le rendement massique (RMba) s'entend ici sur base anhydre, incuits (ou fumerons) déduits, tant de la charge de bois initiale que du produit (charbon de bois et incuits); il est exprimé par l'équation (1).

Les techniques de carbonisation en meule sont comparées dans une revue basée sur l'analyse de plus de 20 publications (Schenkel et al., 1997). Les auteurs concluent que ces techniques, si elles sont bien menées, atteignent des rendements similaires à ceux obtenus par les techniques améliorées des fours métalliques et en maçonnerie.

L'analyse du tableau I met en évidence la très forte variabilité des rendements massiques de carbonisation rapportés dans la littérature, quelle que soit la technique utilisée. Les rendements suivants sont le plus couramment retenus : meules en terre, de 20 à $30 \%$; fours en briques, de 13 à $32 \%$ (la majeure partie supérieure à $25 \%$ ); fours métalliques, de 20 à $25 \%$; fours cornues, $35 \%$ (Schenkel et al., 1997, 1999).

L'évaluation qualitative d'une carbonisation artisanale se base principalement sur le rendement et sur la qualité du charbon de bois produit : taux de matières volatiles, de carbone fixe et de cendres. Ils sont influencés par de nombreux facteurs.

Les premiers ont trait à la matière à carboniser. L'humidité du bois conditionne le rendement massique de carbonisation ; l'eau contenue dans le bois doit être évaporée avant que ne débute la carbonisation. Dans les systèmes à combustion partielle de la charge, cette énergie provient de la combustion de la charge elle-même. La température conditionne aussi le rendement de carbonisation et les propriétés du charbon obtenu. De plus, la masse volumique du bois à carboniser conditionne celle du charbon produit (Doat et Petroff, 1975 ; Girard, 1992 ; Shah et al., 1992).

Ensuite, le rendement de carbonisation est dépendant du savoir-faire du charbonnier. Des aspects tels que le choix du site de carbonisation, la sélection des bois à carboniser (essence, diamètre), l'arrangement des bois ou la constitution d'une sole d'aération sont, en effet, dépendants de l'expérience de l'opérateur. En outre, la carbonisation en meule nécessite une présence permanente du charbonnier auprès de la meule. Après allumage, le charbonnier expérimenté conduit sa meule, c'est-à-dire qu'en gérant les accès d'air (ouverture et fermeture des évents) il assure une progression homogène du front de carbonisation (Sanogo et al., 2006). Sa présence est encore requise en cas d'effondrement de la meule : la carbonisation s'accompagne d'une réduction de volume qui conduit la charge à s'affaisser, ce qui provoque des entrées d'air non contrôlées et engendre la combustion d'une partie parfois importante de la charge, si le charbonnier n'est pas présent pour combler les ouvertures.
La durée d'un cycle de carbonisation en meule varie de 4 à 45 jours, pour des volumes de bois à carboniser qui peuvent varier de 16 à 40 stères (Schenkel et al., 1997). Volume de meule et délai de carbonisation ne sont cependant pas directement liés. D'autres facteurs tels que l'essence de bois, son humidité ou le type de meule utilisée sont à prendre en compte également pour expliquer la durée d'une carbonisation.

Les émissions liées à la carbonisation se répartissent en trois catégories : les gaz non condensables $\left(\mathrm{CO}_{2}, \mathrm{CO}, \mathrm{CH}_{4}\right.$, $\mathrm{H}_{2}, \mathrm{C}_{2} \mathrm{H}_{4}, \mathrm{C}_{2} \mathrm{H}_{6}$ ), les gaz condensables et/ou leurs condensats et les particules. Les proportions dans lesquelles sont émis ces différents gaz dépendent principalement de la température de carbonisation. Le type de four ou la méthode de carbonisation utilisée influencent aussi les émissions finales. En particulier, le principe des fours cornues est de réinjecter les gaz de carbonisation dans le foyer et d'en assurer la combustion pour alimenter le procédé en chaleur. Ceci diminue drastiquement les émissions liées à la carbonisation. Le tableau II reprend les résultats cités dans Schenkel (1999) et compare les émissions (par tonne de charbon de bois) générées par deux procédés de carbonisation différents ; le premier est un système à combustion partielle de la charge, le second est un four cornue.

Des travaux plus récents confirment que les émissions liées à la carbonisation contiennent du méthane. Ce gaz a un impact important sur l'effet de serre (potentiel de réchauffement global à 100 ans - GWP100) 28 fois supérieur à celui du $\mathrm{CO}_{2}$ (Stocker et al., 2013). Les émissions de méthane issues de meules traditionnelles en terre ont été mesurées au Kenya et au Brésil ; elles varient entre 32 et $62 \mathrm{~kg}$ de $\mathrm{CH}_{4}$ par tonne de charbon de bois produite (Pennise et al., 2001). Une autre étude, au Kenya, mentionne des émissions de 32,2 à 46,2 kg de méthane par tonne de charbon de bois produite (Sjølie, 2012). Les émissions en méthane liées à l'utilisation du four Adam ont été estimées à près de $20 \mathrm{~kg}$ de méthane par tonne de charbon de bois produite (Sparrevik et al., 2015). Lors de l'analyse du cycle de vie de différentes filières d'approvisionnement de la sidérurgie brésilienne en charbon de bois, les auteurs évaluent l'émission de méthane, par les fours demi-orange brésiliens, à hauteur de 1,58 Teq $\mathrm{CO}_{2}$ par tonne de charbon de bois (Piketty et al., 2011), soit 75,2 kg de méthane. Des valeurs de 37 à 54 kg de méthane par tonne de charbon de bois produite ont également été citées pour le four Missouri (Riuji Lohri et al., 2016). La méthodologie des mécanismes de développement propre (MDP) utilisée pour calculer la réduction des émissions grâce à l'amélioration de la production et de la consommation de charbon de bois considère une valeur par défaut de $30 \mathrm{~kg}$ de méthane par tonne de charbon de bois (AMS-III.BG Version 3.0, 2013). Ceci correspond à des émissions de carbone liées à la carbonisation, constituées à 2,4\% de méthane si le rendement de carbonisation est de $25 \%$.

Les gaz tels que le $\mathrm{CO}_{2}$ et le $\mathrm{CH}_{4}$ sont donc connus pour leur impact sur l'effet de serre. Si l'on peut considérer que les émissions de $\mathrm{CO}_{2}$ sont d'origine renouvelable et ne contribuent pas fortement au réchauffement climatique, la réduc- 
tion des émissions de méthane est un enjeu majeur de la lutte contre le changement climatique.

Les émissions de gaz à effet de serre liées à la carbonisation peuvent être estimées sur la base du rendement massique (Girard, 2002 ; Girard et Napoli, 2005). Le raisonnement consiste à calculer quelles seraient les émissions carbonées évitées si une technique de carbonisation au rendement massique sur base anhydre (RMba) de $12 \%$ était remplacée par une technique au rendement supérieur. Le rendement de référence considéré est très faible mais plausible ; il correspond au rendement le plus bas relevé par Schenkel et al. (1997) et est également mentionné dans des publications relatives à la carbonisation à Madagascar (Burny et al., 2008 ; Montagne et al., 2010). Les calculs liés à ce raisonnement sont présentés dans le tableau III et illustrés dans la figure 1.

Il est considéré une tonne de bois anhydre dont le contenu en carbone est de $50 \%$. Elle contient donc $500 \mathrm{~kg}$ de carbone. Après carbonisation, une partie de ce carbone demeure dans le charbon de bois ; elle est fonction du rendement massique de carbonisation et de la teneur en carbone fixe (TCF) du charbon de bois. La différence entre le contenu initial en carbone dans le bois et le contenu en carbone dans le charbon de bois représente les émissions de carbone. Dans une première approche, il est considéré que le carbone est intégralement transformé en $\mathrm{CO}_{2}$.

Ainsi, une tonne de bois anhydre carbonisée avec un rendement massique de $12 \%$ produit $120 \mathrm{~kg}$ de matière carbonisée qui contiennent $108 \mathrm{~kg}$ de carbone (dans l'hypothèse d'un TCF de 90 \%). Ce sont donc 392 kg de carbone, par tonne de matière première, qui ont été émis au cours de la carbonisation, ou $3267 \mathrm{~kg}$ de carbone par tonne de charbon de bois. Le même raisonnement mené pour des rendements de $15,20,25,30$ et $35 \%$ conduit à des émissions respectives de 2 433, $1600,1100,767$ et $529 \mathrm{~kg}$ de carbone par tonne de charbon de bois. Par différence avec les émissions liées au rendement de référence et tenant compte d'un rapport de masse $\mathrm{CO}_{2} / \mathrm{C}$ de 3,66 , il est donc estimé que l'utilisation de techniques de carbonisation au rendement massique de $15,20,25,30$ et $35 \%$ en remplacement d'une technique au rendement de $12 \%$ permet d'éviter, au

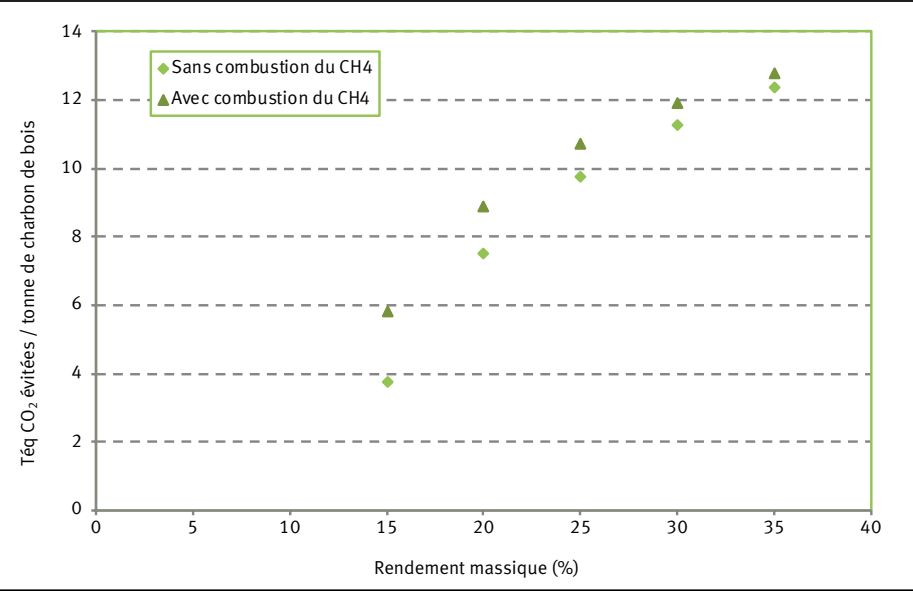

Figure 1.

Tonnes équivalent $\mathrm{CO}_{2}$ évitées grâce à une amélioration du rendement et à une combustion du méthane (par rapport à un rendement massique de carbonisation de référence de $12 \%$ ). minimum et respectivement, $3,06,6,11,7,94,9,16$ et 10,03 Teq $\mathrm{CO}_{2}$ par tonne de charbon de bois.

Or, il a été montré ci-dessus que les émissions des gaz à effet de serre liées à la carbonisation ne se limitent pas au $\mathrm{CO}_{2}$, mais contiennent également du méthane. Le même raisonnement peut être tenu en considérant que les émissions de carbone liées à la carbonisation contiennent 2,5\% de méthane et que son impact sur l'effet de serre est 28 fois supérieur à celui du $\mathrm{CO}_{2}$ (1 tonne de $\mathrm{CH}_{4}=28 \mathrm{Teq} \mathrm{CO}_{2}$ ). Le rapport $\mathrm{CH}_{4} / \mathrm{C}$ est de 1,33 . Selon ces hypothèses, les émissions issues d'une carbonisation au rendement massique de $12 \%$ sont de $81,7 \mathrm{~kg}$ de carbone émis sous forme de $\mathrm{CH}_{4}$ (3,049 Teq $\left.\mathrm{CO}_{2}\right)$ et $3185 \mathrm{~kg}$ de $\mathrm{C}$ émis sous d'autres formes assimilées à du $\mathrm{CO}_{2}\left(11,678 \mathrm{Teq} \mathrm{CO}_{2}\right)$, soit un total de $14727 \mathrm{Teq} \mathrm{CO}_{2}$ par tonne de charbon de bois. Le même raisonnement appliqué aux rendements de carbonisation de $15,20,25,30$ et $35 \%$ conduit à estimer les émissions liées à ces rendements à $10,970-7,213-4,959-3,456$ et 2,383 Teq $\mathrm{CO}_{2}$ par tonne de charbon de bois, soit des émissions évitées de 3,8 - 7,5 - 9,8 - 11,3 et 12,3 Teq $\mathrm{CO}_{2}$ par tonne de charbon de bois, respectivement. Si le méthane est éliminé par le procédé de carbonisation (comme c'est le cas pour les fours cornues), les émissions évitées sont alors de 5,8, 8,9, $10,7,11,9$ et 12,8 Teq $\mathrm{CO}_{2}$ par tonne de charbon de bois.

Dans la limite des hypothèses qui ont été posées, l'analyse de la figure 1 illustre que toute amélioration du rendement de carbonisation conduit à une réduction des gaz à effet de serre. À titre d'exemple, la seule amélioration du rendement de 12 à $15 \%$ permet d'éviter l'émission de près de $4 \mathrm{Teq} \mathrm{CO}_{2}$ dans l'atmosphère, par tonne de produit. Si le méthane émis lors de cette carbonisation peut être éliminé par combustion, le bénéfice environnemental est de 5,8 $\mathrm{Teq} \mathrm{CO}_{2}$. En pratique, cependant, il est peu probable de pouvoir éliminer les émissions de méthane dans des systèmes au rendement inférieur à $25 \%$. En effet, les techniques caractérisées par des rendements faibles ne canalisent généralement pas les gaz issus de la carbonisation et ces derniers sont émis de manière diffuse, ce qui rend improbable toute tentative de combustion. Au-delà d'un rendement de $25 \%$, l'élimination du $\mathrm{CH}_{4}$ peut s'ajouter au bénéfice lié au rendement. Ainsi, l'utilisation d'un four au rendement massique de $35 \%$, qui permet l'élimination du méthane, évitera l'émission de 12,8 $\mathrm{Teq}^{\mathrm{CO}_{2}}$ par tonne de charbon de bois produite, s'il est utilisé en remplacement d'une carbonisation au rendement de $12 \%$.

Ce raisonnement montre que l'amélioration du rendement des procédés de carbonisation et la possibilité d'éliminer les émissions de méthane sont d'une importance primordiale du point de vue environnemental.

C'est pour améliorer ces deux aspects qu'un four de carbonisation à chambre de combustion externe (Green Mad Retort ou GMDR) a été développé pour l'association de charbonniers du village d'Ankitsaka dans la région Diana à Madagascar. La présente étude compare les résultats obtenus avec deux versions de ce four (GMDR 2 et GMDR 3), décrites ci-dessous. Les principales différences entre les deux versions résident dans la complexité et le coût de construction, ainsi que dans la possibilité d'éliminer les gaz de pyrolyse tout au long du procédé. 
Tableau I.

Techniques de carbonisation, synthèse bibliographique des avantages, inconvénients et rendements annoncés.

Technique

\section{Avantages}

(Schenkel, 1999)

\section{Inconvénients}

(Schenkel, 1999)

\section{Rendements} RMba (\%)
Sources

\section{à combustion partielle de la charge}

\begin{tabular}{|c|c|}
\hline & \\
\hline Meule & $\begin{array}{l}\text { Mobilité (tout terrain) } \\
\text { Pas de débardage } \\
\text { Matériaux locaux } \\
\text { Investissement nul } \\
\text { Carbonise les gros bois sans refente } \\
\text { Capacité ajustable } \\
\text { Utilisation de résidus de biomasse }\end{array}$ \\
\hline
\end{tabular}

Exigeant en qualification de l'opérateur

Nécessite beaucoup de main-d'œuvre

(permanente)

Sensible aux aléas climatiques

Charbon de qualité variable et souillé

par la couverture

Rendement énergétique faible

Pollution importante
Traditionnelle

12-34

26

$15-30$

15-35

10-22

9-30

$8-12^{*}$

$10^{*}$

Améliorée

19-42

27

22-34

$18-20^{*}$

$18-20^{*}$

Casamançaise

19-34

25

37

15-30

$15-31$

$17-30$

Fosse

\section{Mobilité}

Débardage sur petit périmètre Matériaux locaux

Investissement nul à très faible

Carbonise les gros bois sans refente

Capacité ajustable

Utilisation de résidus de biomasse

Fours en Matériaux locaux

maçonnerie Conduite aisée

Charbon homogène et propre

Bonne isolation thermique

Peu sensible aux aléas climatiques

Longue durée de vie

Subri

22-36

30

$12-30$

Demi-orange

20

13-32

27-35

Mineirinho

25
Exigeant en qualification de l'opérateur Nécessite beaucoup de main-d'œuvre (permanente)

Sensible aux aléas climatiques

Exige un sol profond et cohérent

Rendement énergétique faible

Pollution importante

Construction nécessitant un maçon

compétent

Refente des gros bois

Capacité déterminée

Problème de coefficient de remplissage

Exigeant en main-d'œuvre

Poussières

Installations fixes

Frais de débardage

Refroidissement lent

Pollution importante (fumées)
22-31

13-35

28-36

Missouri

30

5-33

Adam retort

30

Non spécifié

27-30

Mark V

12-32

20-31

Magnien

25

Non spécifié

21

28

20-38

Pollution imporgente (fumées)

\section{Fours cornues}

Métallique Qualité maîtrisée et homogène Rendement massique élevé Rendement énergétique élevé Pollution faible à nulle Investissement moyen Technicité moyenne

\section{Fours}

industriels

continus à

recirculation

des gaz
Qualité maîtrisée et homogène

Rendement massique élevé

Rendement énergétique élevé

Pollution faible à nulle

Automatisation
Transport du bois (sauf pour les

cornues mobiles)

Refente et préparation du bois

Capacité déterminée

Problèmes de coefficient de remplissage

Nécessité de séchage du bois Déformation des enceintes

\section{Investissement considérable}

26-35

Technicité élevée

30-35

23-32

$22-40$

Périmètre d'approvisionnement étendu

Transport du bois

Refente et préparation du bois
Schenkel et al., 1997

Girard, 1992

Sanogo et al., 2006

Riuji Lohri, 2016

Madon, 2017

FAO, 2017

Montagne et al., 2010

Burny et al., 2008

Schenkel et al., 1997

Mundhenk et al., 2010

Sjolie, 2012

Montagne et al., 2010

Burny et al., 2008

Schenkel et al., 1997

Girard, 1992

Mundhenk et al., 2010

Sanogo et al., 2006

Riuji Lohri, 2016

FAO, 2017

Schenkel et al., 1997

Schenkel et al., 1999

FAO, 2017

Piketty et al., 2011

Schenkel et al., 1997

FAO, 2017

Piketty et al., 2011

Schenkel et al., 1997

Riuji Lohri, 2016

Pennise, 2001

Piketty et al., 2011

Riuji Lohri C., 2016

Sparrevik et al., 2015

Schenkel et al., 1999

Schenkel et al., 1997

Riuji Lohri et al., 2016

Girard, 1992

Schenkel et al., 1999

Schenkel et al., 1999

FAO, 2017

Schenkel et al., 1999

FAO, 2017

Schenkel et al., 1999

Riuji Lohri et al., 2016

* Données mentionnées parce que récoltées à Madagascar, cependant type de rendement et humidité non spécifiés. 


\section{Tableau II.}

Impact environnemental de la carbonisation : principaux polluants atmosphériques pour deux systèmes de carbonisation, exprimés en $\mathrm{kg} /$ tonne de charbon de bois, d'après Schenkel (1999). COV : composés organiques volatils; HCT : hydrocarbures totaux ; HAP : hydrocarbures aromatiques polycycliques.

\begin{tabular}{l|c|c|} 
& Four à combustion partielle & Four cornue industriel \\
\hline Poussières et vésicules & 32 & 3 \\
\hline CO & 340 & 12 \\
\hline COV (hors méthane) & 100 & 9,5 \\
\hline HCT (hors méthane) & 40 & 0 \\
\hline Méthane & 40 & 0 \\
\hline Phénols & 0,6 & 0,2 \\
\hline Acide acétique & 48 & 4,5 \\
\hline Méthanol & 8 & 0,75 \\
\hline Acide formique & 10 & 0,85 \\
\hline Acide propionique & 4 & 0,15 \\
\hline Furfural & 5 & 0,5 \\
\hline HAP & 1,35 & 0 \\
\hline
\end{tabular}

dans la cheminée d'évacuation des gaz provenant de la chambre de carbonisation.

Après chargement et fermeture du four, la première étape est d'allumer un feu dans la chambre de combustion $\left(n^{\circ} 1\right)$, les gaz chauds qu'il génère sont conduits dans le canal $\left(n^{\circ} 3\right)$ sous la charge, vers la cheminée d'évacuation des gaz de combustion $\left(n^{\circ} 4\right)$. Ce faisant, la charge est chauffée et l'air chaud qu'elle contient commence à s'évacuer par l'autre cheminée $\left(n^{\circ} 6\right)$; l'entrée de cette cheminée se situe en effet dans la chambre de carbonisation. Un flux d'air est donc généré dans la chambre de carbonisation : de l'air chaud en provenance de la chambre de combustion ( $n^{\circ}$ 2) s'introduit dans la chambre de carbonisation, se charge en vapeur d'eau issue du bois, puis est évacué par la cheminée reliée à la chambre de carboni-

\section{Matériels et méthodes}

\section{Les fours de carbonisation étudiés}

\section{GMDR 2}

Le four Green Mad Retort 2 est constitué de six parties principales: une chambre de combustion externe $\left(n^{\circ} 1\right.$ sur la figure 2), une connexion (tuyaux) entre la chambre de combustion et la chambre de carbonisation ( $\left.n^{\circ} 2\right)$, une chambre de carbonisation (entre $n^{\circ} 2$ et $n^{\circ} 5$ ), un canal d'évacuation des gaz de combustion situé sous la chambre de carbonisation ( $n^{\circ} 3$ ) et, enfin, deux cheminées, la première ( $\left.{ }^{\circ} 4\right)$ étant reliée au canal d'évacuation des gaz de combustion et la seconde à la chambre de carbonisation ( $n^{\circ}$ 6). La chambre de carbonisation et le canal d'évacuation des gaz sont séparés par une tôle métallique. Ces différents éléments sont également illustrés par la figure 3. Des thermomètres permettent de mesurer la température des gaz dans le haut et le bas de la chambre de carbonisation, ainsi que

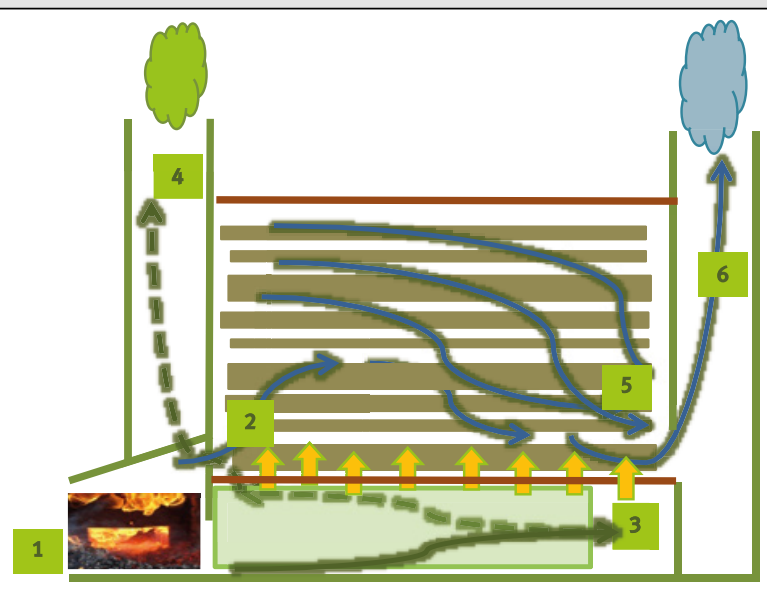

Figure 2.

Le four GMDR 2 : principe de fonctionnement. sation. C'est la phase de séchage, dont la durée varie de 12 à 36 heures en fonction de l'humidité de départ du bois. Durant cette phase, la température ne dépasse pas $100^{\circ} \mathrm{C}$.

Lorsque l'eau a quitté le bois, celui-ci monte en température pour atteindre les niveaux qui permettent la carbonisation $\left(280^{\circ} \mathrm{C}\right.$ et au-delà). Les gaz émis durant cette phase sont combustibles. Lorsque les températures dans les parties inférieures et supérieures du four sont égales, la cheminée d'évacuation des gaz de la chambre de carbonisation est bouchée. La seule échappatoire des gaz est alors constituée par les tubulures reliant la chambre de carbonisation et la chambre de combustion. Arrivant dans la chambre de combustion, les gaz entrent en contact avec l'air et s'enflamment violemment. Cette phase perdure 3 à 6 heures et se termine avec la carbonisation. Tous les accès d'air du four sont bouchés et le four est laissé à refroidir pendant 3 ou 4 jours.

Dans les conditions malgaches, les coûts de production d'un tel four, hors accompagnement et formation à l'utilisation, sont de l'ordre de 5000 à $6000 €$.

\section{GMDR 3}

La GMDR 3 est très similaire à la GMDR 2, mais la tôle de fond est supprimée, de même que le système de canaux la soutenant et la cheminée qui évacue les gaz passant par ces canaux, sous la tôle. La construction est donc simplifiée, ce qui permet de réduire les coûts à $3000 €$ environ.

Le principe de fonctionnement de la GMDR 3 est illustré par la figure 4.

Une ouverture est pratiquée à la base de l'unique cheminée du four ; elle a pour but d'y allumer un feu qui génère un tirage au niveau des tuyaux entre les chambres de combustion et de carbonisation, lors de l'allumage. Lorsque le flux est amorcé, il s'autoalimente et le feu à la base de la cheminée n'est plus nécessaire. Il est à nouveau allumé lors 
Tableau III.

Évolution des tonnes équivalent $\mathrm{CO}_{2}\left(\mathrm{Teq}_{\mathrm{CO}}\right.$ ) minimums évitées par une amélioration du rendement de carbonisation, exprimées par tonne de charbon de bois produite ( $\mathrm{TPd}$ ). Les estimations réalisées selon l'approche 2 considèrent l'impact du $\mathrm{CH}_{4}$, celles de l'approche 1 le négligent - rapport $\mathrm{CO}_{2} / \mathrm{C}=3,66$; rapport $\mathrm{CH}_{4} / \mathrm{C}=1,33 ; 1$ tonne $\mathrm{CH}_{4}=28 \mathrm{Teq}_{2}$.

\begin{tabular}{|c|c|c|c|c|c|c|c|}
\hline \multirow{2}{*}{$\begin{array}{l}\text { Système de carbonisation } \\
\text { Masse de bois anhydre }\end{array}$} & \multirow{2}{*}{$\begin{array}{l}\text { Unités } \\
\text { kg }\end{array}$} & \multirow{2}{*}{$\begin{array}{l}\text { CBR } \\
1000\end{array}$} & & \multicolumn{4}{|c|}{ Rendements de carbonisation croissants } \\
\hline & & & 1000 & 1000 & 1000 & 1000 & 1000 \\
\hline Taux de carbone dans le bois & $\%$ & 50 & 50 & 50 & 50 & 50 & 50 \\
\hline Masse de carbone dans le bois & $\mathrm{kg}$ & 500 & 500 & 500 & 500 & 500 & 500 \\
\hline Rendement de carbonisation & $\%$ & 12 & 15 & 20 & 25 & 30 & 35 \\
\hline Masse de charbon de bois & $\mathrm{kg}$ & 120 & 150 & 200 & 250 & 300 & 350 \\
\hline Taux C du charbon de bois & $\%$ & 90 & 90 & 90 & 90 & 90 & 90 \\
\hline Masse $\mathrm{C}$ du charbon de bois & $\mathrm{kg}$ & 108 & 135 & 180 & 225 & 270 & 315 \\
\hline Émissions C de la conversion & $\mathrm{kg}$ & 392 & 365 & 320 & 275 & 230 & 185 \\
\hline Émissions spécifiques & $\mathrm{kg} \mathrm{C} / \mathrm{TPd}$ & 3267 & 2433 & 1600 & 1100 & 767 & 529 \\
\hline Émissions évitées & $\mathrm{kg} \mathrm{C} / \mathrm{TPd}$ & & 833 & 1667 & 2167 & 2500 & 2738 \\
\hline $\begin{array}{l}\text { Réduction minimale des GES } \\
\text { (approche 1) }\end{array}$ & $\mathrm{Teq} \mathrm{CO}_{2} / \mathrm{TPd}$ & 0 & 3,06 & 6,11 & 7,94 & 9,16 & 10,03 \\
\hline $\begin{array}{l}\text { Teneur en } \mathrm{CH}_{4} \text { des fumées } \\
\text { de carbonisation }\end{array}$ & $\%$ & 2,5 & 2,5 & 2,5 & 2,5 & 2,5 & 2,5 \\
\hline $\begin{array}{l}\text { Émissions spécifiques } \mathrm{C} \\
\text { sous forme de } \mathrm{CH}_{4}\end{array}$ & $\mathrm{~kg} \mathrm{C} / \mathrm{TPd}$ & 81,7 & 60,8 & 40,0 & 27,5 & 19,2 & 13,2 \\
\hline $\begin{array}{l}\text { Émissions spécifiques C } \\
\text { sous une autre forme }\end{array}$ & $\mathrm{kg} \mathrm{C} / \mathrm{TPd}$ & 3185 & 2373 & 1560 & 1073 & 748 & 515 \\
\hline Impact $\mathrm{CH}_{4}$ & $\mathrm{Teq} \mathrm{CO}_{2} / \mathrm{TPd}$ & 3,049 & 2,271 & 1,493 & 1,027 & 0,716 & 0,493 \\
\hline Impact des autres composés & $\mathrm{Teq} \mathrm{CO}_{2} / \mathrm{TPd}$ & 11,678 & 8,699 & 5,720 & 3,933 & 2,741 & 1,890 \\
\hline Impact de la carbonisation & $\mathrm{Teq} \mathrm{CO}_{2} / \mathrm{TPd}$ & 14,727 & 10,970 & 7,213 & 4,959 & 3,456 & 2,383 \\
\hline $\begin{array}{l}\text { Émissions évitées (approche } 2 \text { - } \\
\text { sans élimination du } \mathrm{CH}_{4} \text { ) }\end{array}$ & $\mathrm{Teq} \mathrm{CO}_{2} / \mathrm{TPd}$ & & 3,8 & 7,5 & 9,8 & 11,3 & 12,3 \\
\hline $\begin{array}{l}\text { Émissions évitées (approche } 2 \text { - } \\
\text { avec élimination du } \mathrm{CH}_{4} \text { ) }\end{array}$ & $\mathrm{Teq} \mathrm{CO}_{2} / \mathrm{TPd}$ & & 5,8 & 8,9 & 10,7 & 11,9 & 12,8 \\
\hline
\end{tabular}
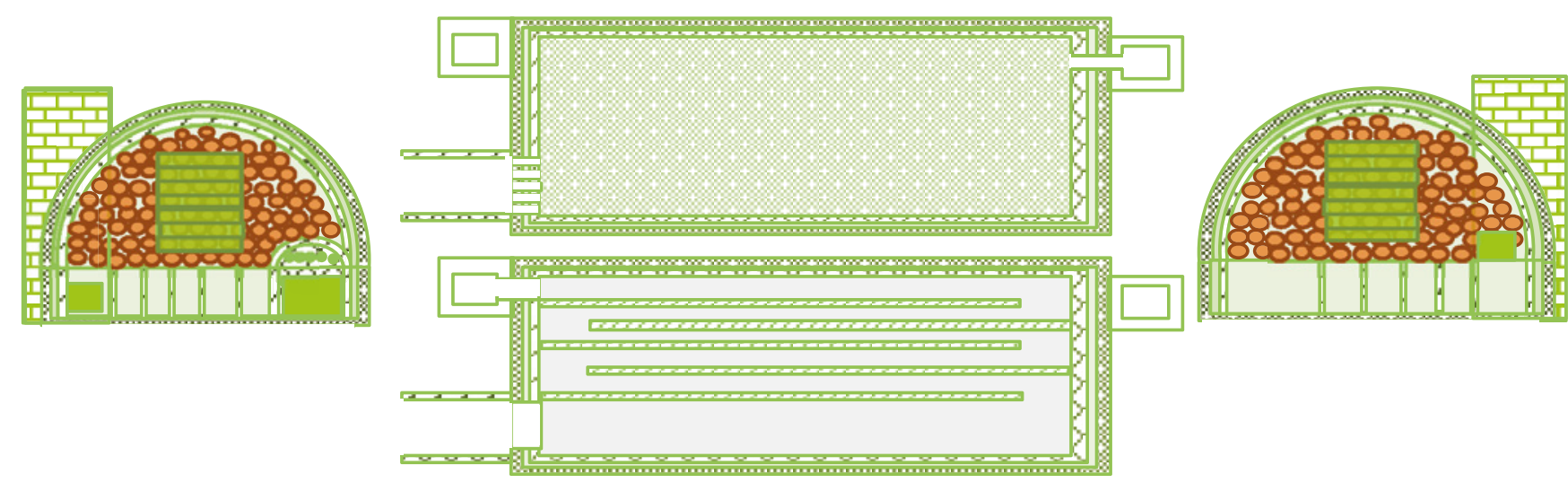

Figure 3.

Le four GMDR 2 : vue en coupe et en élévation. 


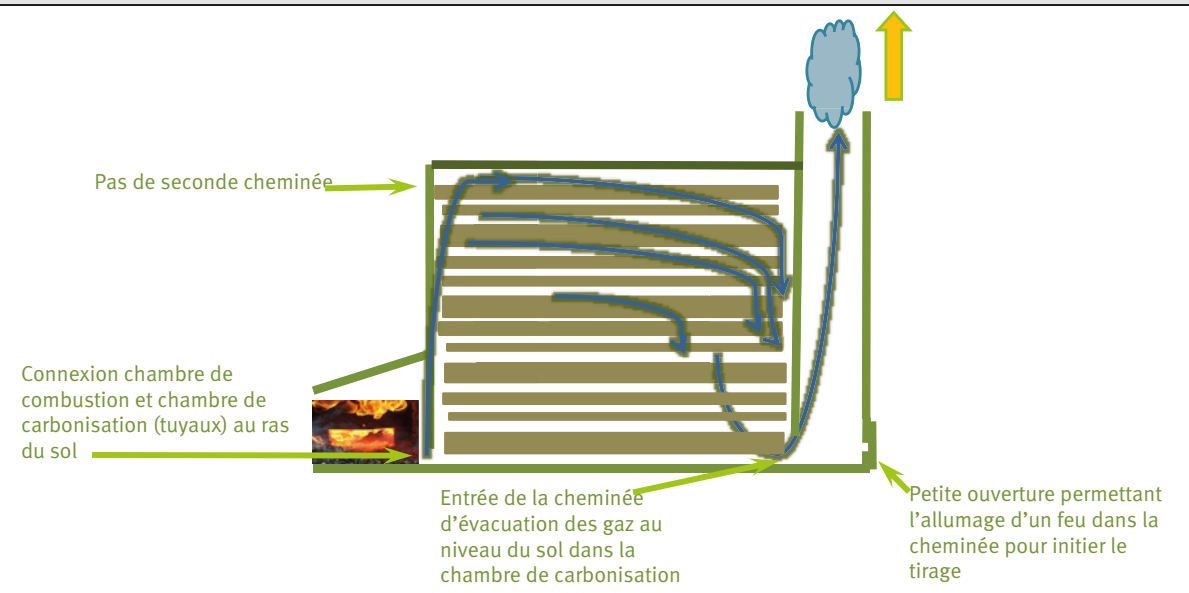

Figure 4.

GMDR 3 : principe de fonctionnement. calyptus camaldulensis Dehnh. de 8 ans, non écorcé, issu des plantations énergétiques aux alentours du village d'Ankitsaka. La possibilité d'utiliser des résidus sans valeur (sous-produits agricoles ou fines branches) comme combustible pour la chambre de combustion externe n'a pas été considérée dans cette étude.

\section{Masse de bois constituant la charge}

La masse de bois humide constituant la charge a été déterminée à l'aide d'un peson numérique d'une capacité maximale de $200 \mathrm{~kg}$ avec une précision de 0,1 kg.

La masse du bois combustible utilisé dans la chambre de combustion a été déterminée également. de la phase de pyrolyse, afin de faciliter la combustion du méthane et d'épurer thermiquement les fumées émises tout au long du procédé.

Lors du chargement des deux fours considérés, les rondins de bois sont disposés dans le sens du flux des gaz et ne sont pas écorcés.

La GMDR 3 est illustrée en photo 1, le charbon produit en photo 2 .

\section{La matière première et le produit}

\section{Essence}

Lors des essais, le bois destiné à la carbonisation et le bois combustible étaient constitués exclusivement d'Eu-

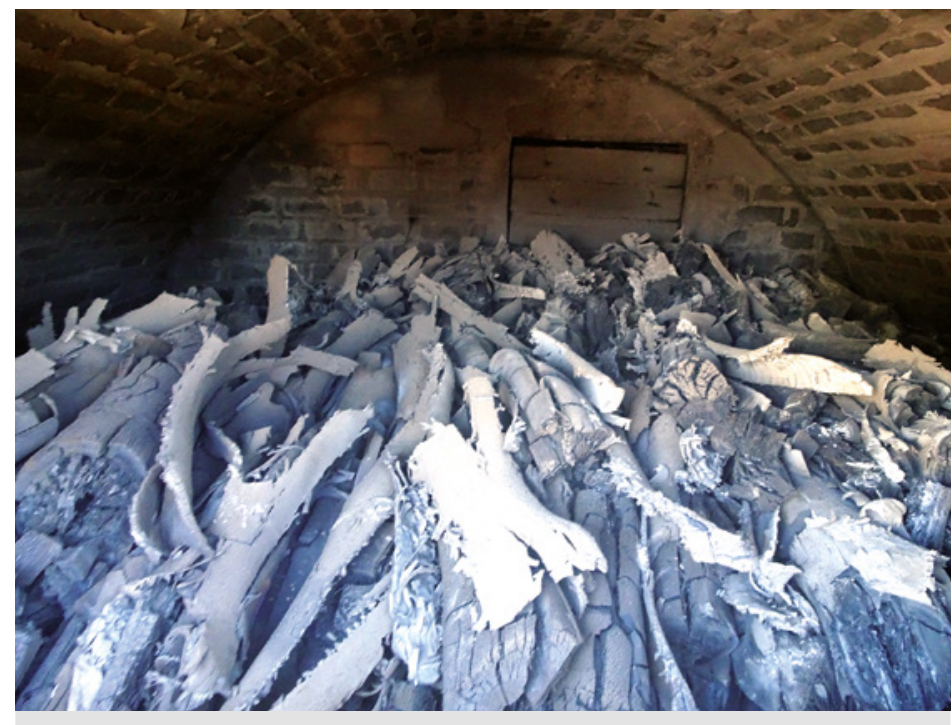

Photo 2.

Vue du charbon de bois contenu dans la GMDR 3 au terme de la carbonisation.

Photo M. Temmerman.

\section{Mesure de l'humidité}

L'humidité du bois et des éventuels incuits a été estimée à l'aide d'un hygromètre électrique (Testo 606-1) dont la précision est de $0,1 \%$. La prise d'humidité a été pratiquée sur le duramen. À cet effet, une encoche a été pratiquée dans les rondins, à la hache. L'hygromètre électrique ne pouvait être utilisé pour la mesure de l'humidité du charbon de bois. Il a donc été considéré que lors des mesures de la masse de charbon produite, quelques heures après le défournement, le charbon de bois s'était équilibré à une humidité de $5 \%$ (ISO 18134-2:2017).

\section{Le charbon de bois}

À l'issue de la carbonisation (après la phase de refroidissement), les produits peuvent être différenciés en trois catégories : le charbon de bois commercialisable, le charbon de moindre valeur (fines et braisettes) et les incuits ou fumerons. Chacune de ces catégories a été pesée séparément à l'aide du même peson qui a été utilisé pour peser la charge.

Lors d'un des essais réalisés sur la GMDR 2, un échantillon de charbon de bois a été prélevé pour l'analyse du pouvoir calorifique supérieur (PCS), des taux de carbone fixe (TCF), de la matière volatile (TMV) et des cendres (TC) du charbon de bois produit, selon les normes ISO (ISO 18122:2015 ; ISO 18123:2015). Pour cet échantillon, les valeurs suivantes ont été obtenues : PCS $=30,7 \mathrm{MJ} / \mathrm{kg} ; \mathrm{TCF}=74,05 \%$; $\mathrm{TMV}=29,95 \% ; \mathrm{TC}=0,44 \%$.

\section{Calcul des rendements}

Les résultats présentés dans cette étude sont basés sur le suivi de 23 carbonisations réalisées dans la GMDR 2 et 25 dans la GMDR 3.

\section{Le rendement massique}

Le rendement massique est calculé selon l'équation (1). Il considère la totalité du charbon de bois (anhydre) produit, quelle que soit sa forme (morceaux ou fines), et la totalité du 
bois anhydre contenu dans la charge (y compris le combustible). La masse anhydre d'incuits est soustraite de la masse anhydre de bois au départ.

$$
R M_{b a}=\frac{M_{c a}}{M_{b a}-M_{i a}} 100
$$

avec RMba : rendement massique sur base anhydre (\%) ; Mca : masse de matière carbonisée anhydre, charbon de bois et fines $(\mathrm{kg})$; Mba : masse de bois anhydre $(\mathrm{kg})$; Mia : masse des incuits anhydres $(\mathrm{kg})$.

\section{Le rendement commercial}

Le rendement commercial se calcule de la même manière que le rendement massique mais considère la masse anhydre de charbon de bois qui peut être commercialisée à partir de la production. Les fines et les braisettes ne sont pas considérées.

\section{Réalisation des mesures}

Les mesures réalisées dans le cadre de cette étude sont des mesures de terrain. Les conditions dans lesquelles elles ont été effectuées ne permettaient pas l'emploi d'équipements de mesure sophistiqués, notamment pour des raisons d'accessibilité du site de carbonisation, d'accès à l'électricité et d'humidité de l'atmosphère. Des méthodes simples et des appareils de mesure portables ont donc été utilisés, tels que décrits ci-après. Les données et résultats présentés ici devront idéalement être confirmés par des essais réalisés dans les

\section{Tableau IV.}

Caractéristiques des carbonisations réalisées

\begin{tabular}{|c|c|c|c|}
\hline & Unités & $\begin{array}{c}\text { GMDR } 2 \\
\text { Moyenne } \\
\text { (écart-type) }\end{array}$ & $\begin{array}{c}\text { GMDR } 3 \\
\text { Moyenne } \\
\text { (écart-type) }\end{array}$ \\
\hline Répétition & $\mathrm{N}$ & 23 & 25 \\
\hline Durée de la carbonisation & $\mathrm{H}$ & $53(14,0)$ & $50(11,6)$ \\
\hline Masse charge & $\mathrm{kg}$ & $3092(307)$ & 3031 (192) \\
\hline Humidité charge & $\%$ & $36(3,5)$ & $36(2,6)$ \\
\hline Masse combustible & $\mathrm{kg}$ & $231(67)$ & $242(66)$ \\
\hline Humidité combustible & $\%$ & $36(3,5)$ & $36(2,6)$ \\
\hline Masse charbon & $\mathrm{kg}$ & $728(94)$ & $675(74)$ \\
\hline Masse poudre & $\mathrm{kg}$ & $69(17)$ & $63(24)$ \\
\hline Masse Incuits & $\mathrm{kg}$ & $1(5)$ & $32(34)$ \\
\hline Masse charge anhydre & $\mathrm{kg}$ & $1992(240)$ & 1944 (145) \\
\hline Masse combustible anhydre & $\mathrm{kg}$ & $148(42)$ & $155(41)$ \\
\hline Masse charbon anhydre & $\mathrm{kg}$ & $692(89)$ & $642(70)$ \\
\hline Masse fines anhydre & $\mathrm{kg}$ & $66(16)$ & $60(23)$ \\
\hline Masse incuits anhydre & $\mathrm{kg}$ & $1(5)$ & $28(30)$ \\
\hline $\mathrm{N}$ sac de charbon & $\%$ & $83(12)$ & $75(7)$ \\
\hline Masse moyenne des sacs & $\mathrm{kg}$ & $8,8(0,4)$ & $9,0(0,5)$ \\
\hline Rendement massique & $\%$ & $35,6(3,2)$ & $33,9(2,4)$ \\
\hline Rendement commercial & $\%$ & $32,4(3,2)$ & $31,0(2,5)$ \\
\hline $\begin{array}{l}\text { Pourcentage combustible } \\
\text { dans masse totale }\end{array}$ & $\%$ & $7,5(2,1)$ & $8,0(2,1)$ \\
\hline
\end{tabular}

conditions standardisées d'un laboratoire. Ils apportent néanmoins un éclairage sur les valeurs obtenues dans les conditions opérationnelles de la carbonisation.

Parmi les carbonisations destinées à l'évaluation des rendements, certaines ont fait l'objet d'une estimation des émissions en $\mathrm{CH}_{4}: 17$ pour la GMDR 2 et 6 pour la GMDR 3 .

\section{Mesure des températures}

Les températures ont été mesurées à l'aide d'un thermomètre Testo équipé de thermocouples type K.

Un thermocouple a été placé dans la cheminée afin de déterminer la température des fumées.

Deux autres thermocouples ont été positionnés à $30 \mathrm{~cm}$ du mur de la cheminée de la chambre de carbonisation, l'un au niveau de la partie supérieure de la voûte, l'autre au niveau du plancher de la GMDR (2 ou 3)

\section{Calcul du débit}

Le débit des fumées a été estimé grâce à un diaphragme inséré dans le conduit de la cheminée. La différence de pression entre l'amont et l'aval du diaphragme est proportionnelle au débit des fumées ; elle a été mesurée grâce à un appareil de mesure de la pression différentielle Testo 510, d'une résolution de 0,01 hPa.

\section{Mesure de la concentration en $\mathrm{CH}_{4}$}

Un détecteur Sewerin PM4 calibré sur le méthane a été utilisé périodiquement (afin d'éviter l'encrassement trop rapide des filtres) pour mesurer la concentration de ce gaz dans les fumées s'échappant de la cheminée. La gamme de mesures s'étendait de 0 à 22000 ppm (2,2 \%) par volume de méthane.

La masse de $\mathrm{CH}_{4}$ émise au cours d'une carbonisation a été approchée en utilisant la loi des gaz parfaits $(P V=n R T)$ où $P$ est la pression du gaz (en pascals), ici considérée comme constante et égale à la pression atmosphérique, $\mathrm{V}$ le volume occupé par le gaz (en $\mathrm{m}^{3}$ ), ici déduit des mesures de débit, $\mathrm{n}$ la quantité de matière (en moles), $\mathrm{R}$ la constante universelle des gaz parfaits $(8,3144621 \mathrm{~J} / \mathrm{K} / \mathrm{mol})$ et $\mathrm{T}$ la température (en kelvins), déduite des mesures des températures.

\section{Périodicité des mesures}

Les mesures ont été réalisées toutes les heures depuis l'allumage du four jusqu'à son colmatage avant la phase de refroidissement. Elles consistaient en l'enregistrement de la température et du débit des fumées, de la température dans les parties haute et basse des GMDR ainsi que de la concentration en méthane des fumées. La durée de ce suivi correspondait également au temps durant lequel les charbonniers devaient être présents sur le site. En effet, leur présence n'était pas requise durant la phase de refroidissement.

À l'exception de la masse de $\mathrm{CH}_{4}$ émise $\mathrm{au}$ cours de la carbonisation, les résultats relatifs au procédé présentés ici se concentrent sur les dernières 48 heures de carbonisation. 


\section{Résultats}

Le tableau IV présente les principales caractéristiques des carbonisations réalisées au cours de cette étude.

Lors des 23 carbonisations réalisées dans la GMDR 2, la masse moyenne de la charge était de 3093 kg à une humidité moyenne de $36 \%$, soit une masse de bois anhydre contenue dans la charge de $1992 \mathrm{~kg}$ en moyenne. Les productions moyennes ont été de $692 \mathrm{~kg}$ de charbon anhydre, $66 \mathrm{~kg}$ de fines et $1 \mathrm{~kg}$ d'incuits. Le charbon a été conditionné, en moyenne, en 83 sacs d'une masse moyenne de $8,8 \mathrm{~kg}$. Ces valeurs permettent de calculer un rendement massique moyen pour les carbonisations pratiquées de 35,6\% et un rendement commercial de $32,4 \%$. Le temps de présence moyen requis pour les charbonniers était de 53 heures.

Lors des 25 carbonisations réalisées dans la GMDR 3, les valeurs moyennes obtenues étaient très proches de celles obtenues avec les GMDR 2. La masse moyenne de la charge était de $3031 \mathrm{~kg}$ à une humidité moyenne de $36 \%$, soit une masse de bois anhydre contenue dans la charge de $1944 \mathrm{~kg}$ en moyenne. Les productions moyennes ont été de $642 \mathrm{~kg}$ de charbon anhydre, $60 \mathrm{~kg}$ de fines et $32 \mathrm{~kg}$ d'incuits. Le charbon a été conditionné, en moyenne, en 75 sacs d'une masse moyenne de 9,0 kg. Ces valeurs permettent de calculer un rendement massique moyen pour les carbonisations pratiquées de 33,9\% et un rendement commercial de $31,0 \%$. Le temps de présence moyen requis pour les charbonniers était de 50 heures.

Les rendements massiques et commerciaux sont illustrés par la figure 5 où il apparaît, d'une part, que les rendements obtenus par les GMDR 2 et 3 sont sensiblement égaux et, d'autre part, que tant les rendements massiques que les rendements commerciaux sont très élevés. Ils se situent dans la fourchette haute des rendements de carbonisation présentés dans le tableau I et sont également très proches des rendements rapportés pour les fours cornues. Il faut également souligner les faibles écarts-types associés à ces moyennes. Ils indiquent la bonne stabilité des rendements obtenus, ce qui contraste avec les autres tech-

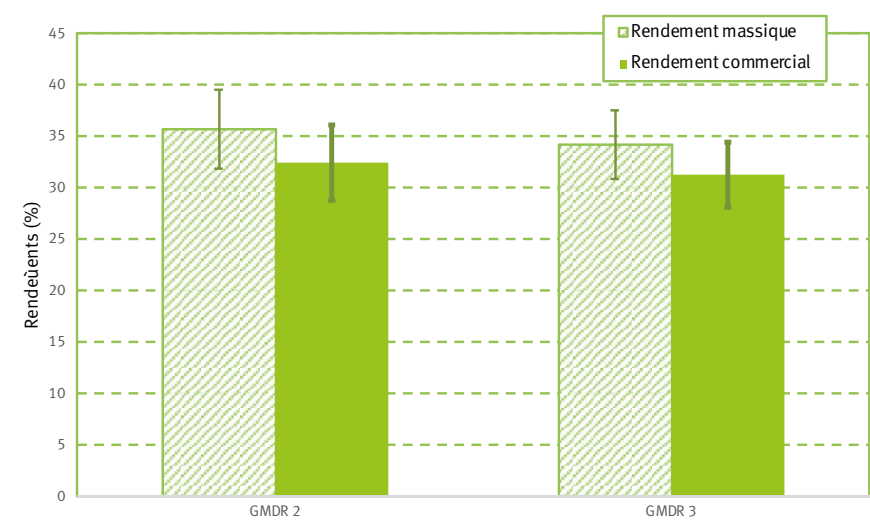

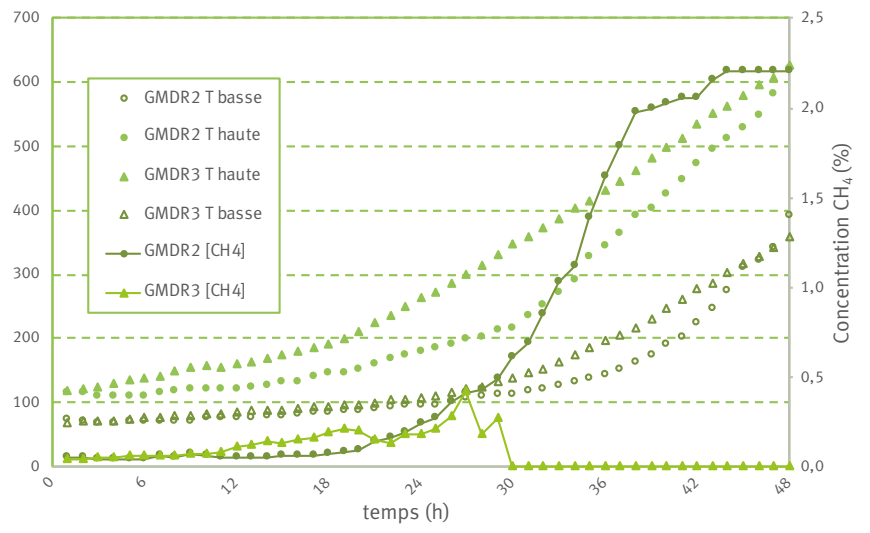

Figure 6.

Évolution de la concentration en méthane dans les fumées $\left(\mathrm{CH}_{4}\right)$ et de la température dans les parties hautes ( $T$ haute) et basses ( $T$ basse) des GMDR, valeur moyenne au cours des dernières 48 heures de carbonisation. (N GMDR $2=17$ et N GMDR $3=6$ )

niques de carbonisation artisanales, en particulier les meules en terre. De même, l'humidité moyenne des charges est relativement élevée (36\%), ce qui semblerait indiquer que cette technique de carbonisation est relativement peu sensible à ce facteur, même si cet aspect devrait être confirmé par une expérimentation spécifique.

D’un point de vue pratique, l'égalité des rendements calculés pour les deux fours confirme la possibilité de simplifier la construction du four et de se passer de la tôle de fond, des canaux et de la seconde cheminée. La construction est donc plus simple et moins coûteuse.

La figure 6 présente les profils moyens de la concentration en méthane dans les fumées, relevés pour les GMDR 2 et 3 au cours des 48 dernières heures de carbonisation. Ces valeurs sont mises en relation avec les températures moyennes relevées au même moment dans les parties hautes et basses des fours. L'évolution des températures est similaire dans les deux fours. Les températures en haut et en bas de la chambre de carbonisation augmentent régulièrement, les premières étant toujours supérieures aux secondes, sauf en fin de processus, quand la température en bas s'élève rapidement pour atteindre des valeurs similaires à celle du haut de la chambre de carbonisation. Cette jonction n'apparaît pas sur la figure 6 qui présente les moyennes de plusieurs mesures. Mais elle est caractéristique des profils de température individuels de chaque carbonisation. C'est en outre le signe qu'attendent les charbonniers pour boucher la cheminée d'évacuation des gaz de carbonisation. L'explication d'un front de carbonisation progressant de haut en bas peut être avancée. La température des fumées émises par la cheminée (non présentée sur la figure 6) est intermédiaire entre la température haute et la température basse ; elle est également constamment croissante.

L'évolution de la concentration en méthane dans les fumées est très différente pour les deux fours testés. Les émissions de la GMDR 2, très faibles en début de carbonisation (elles sont de l'ordre du ppm durant la phase séchage), augmentent progressivement pour

Figure 5.

Rendements massiques (moyenne et écart-type). 


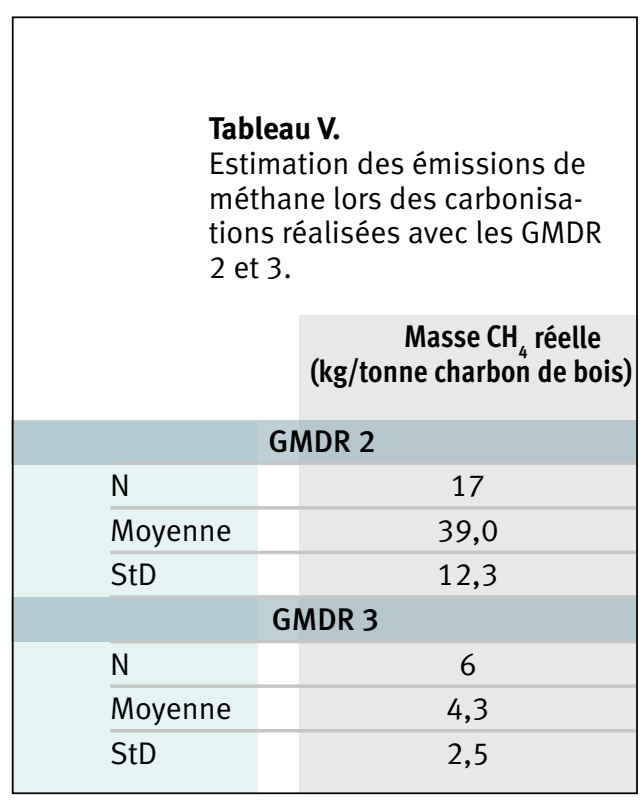

atteindre des valeurs de 1 à $2 \%$ et davantage en fin de carbonisation.

Lors de l'utilisation de la GMDR 3, il est apparu que les fumées étaient combustibles à partir d'une concentration en méthane de $0,5 \%$. Par suite de cette épuration thermique, la concentration en méthane des gaz s'échappant de la cheminée est nulle.

Outre les mesures réalisées, l'effet qualitatif est manifeste : les fumées, jaunâtres, caractéristiques des carbonisations à combustion partielle de la charge, disparaissent pour laisser place à un flux gazeux transparent. Cet effet

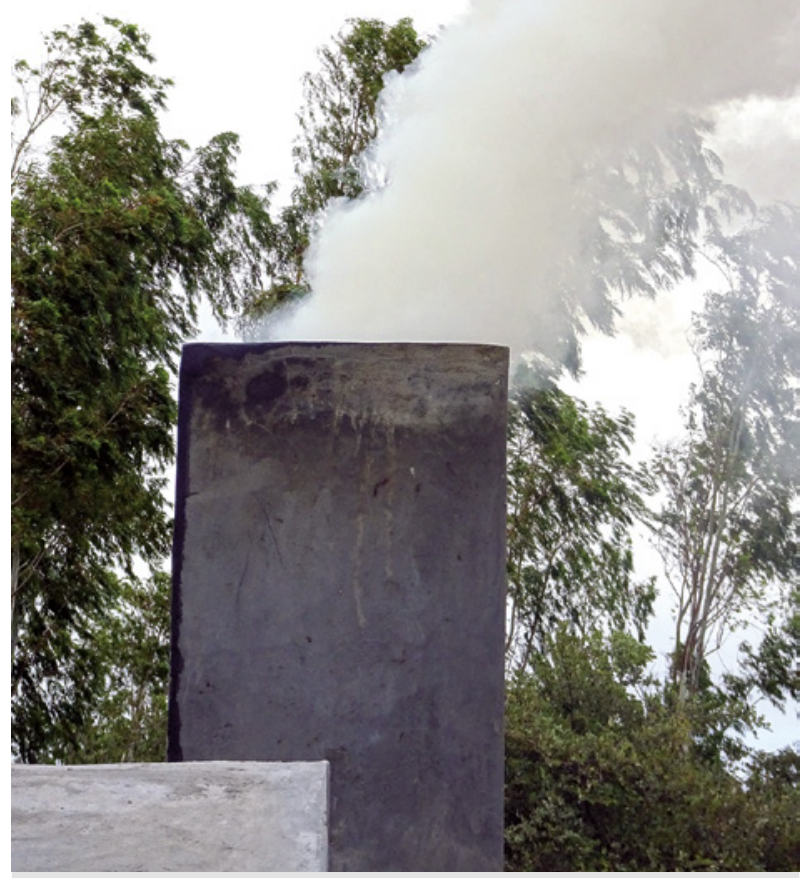

Photo 3.

Fumées en phase de pyrolyse (sans épuration des fumées). Photo M. Temmerman.

qualitatif est observable sur les photos 3 et 4.

Les concentrations en méthane présentées à la figure 6 ont été converties en masse et sont présentées dans le tableau V. Les émissions liées à une carbonisation sans élimination du méthane (GMDR 2) sont estimées à $39 \mathrm{~kg}$ par tonne de charbon de bois produite. Elles tombent à 4,3 kg par tonne de charbon de bois pour la GMDR 3. Comparée aux valeurs publiées jusqu'à présent pour les techniques de carbonisation artisanales (20 à $75 \mathrm{~kg}$ de méthane par tonne de charbon de bois), cette dernière valeur constitue une réduction d'un facteur 5 à 17. Ce niveau d'émission semble n'avoir été atteint jusqu'à présent que par les fours cornues industriels. Dans les hypothèses de la figure 1 , ces valeurs indiquent que l'utilisation de la GMDR permettrait d'éviter l'émission de près de 13 Teq $\mathrm{CO}_{2}$ par tonne de charbon de bois produite, par rapport à une carbonisation à faible rendement.

Ces résultats issus de mesures de terrain seront idéalement confirmés par l'utilisation d'équipements permettant des mesures en continu et sur une gamme de valeurs plus étendue.

La GMDR présente aussi l'avantage d'un procédé rapide et séquencé. La durée de carbonisation proprement dite est d'une cinquantaine d'heures, auxquelles il faut ajouter trois jours de refroidissement durant lesquels la présence des charbonniers n'est pas requise. Un cycle de carbonisation produit environ $650 \mathrm{~kg}$ de charbon de bois. Le temps que les charbonniers doivent consacrer à leur activité est donc réduit par rapport, par exemple, aux meules en terre. En effet, la carbonisation de la même quantité de bois dans une meule en terre peut nécessiter jusqu'à une dizaine de jours de présence sur place.

La durée de vie d'une GMDR doit aussi être mentionnée. La première GMDR, construite en 2012 à Ankitasaka, à Madagascar, est toujours en activité après environ 250 cycles de carbonisation.

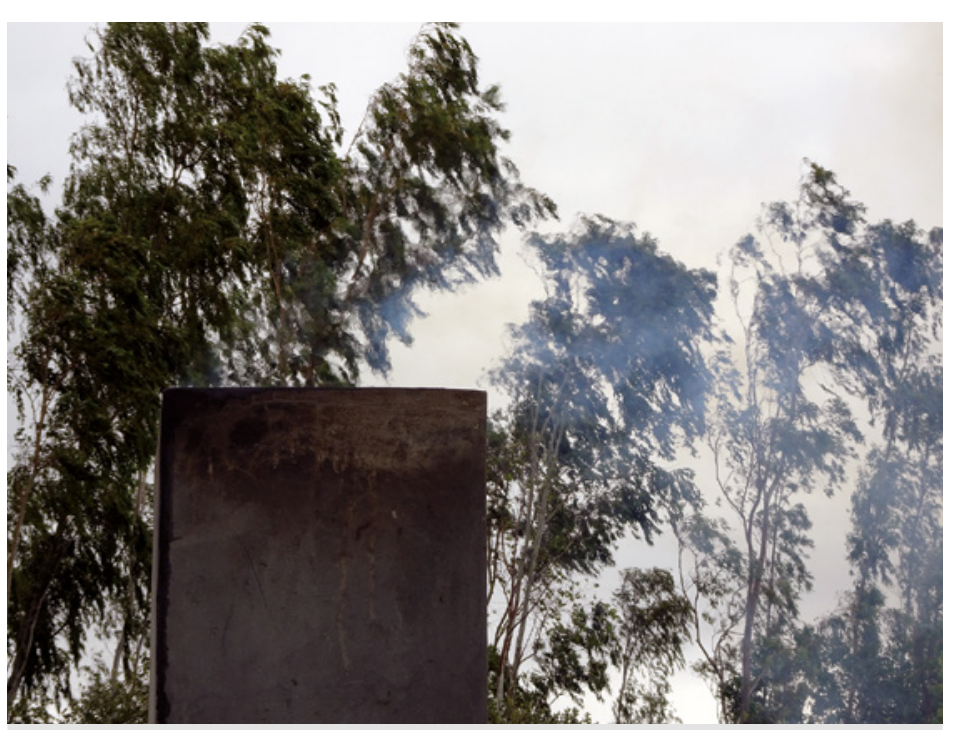

Photo 4. 


\section{Conclusion}

À Madagascar, la GMDR, ou Green Mad Retort, est une technologie qui s'affiche comme une technique de production du charbon de bois à haut rendement. La présente étude a été menée sur ce four de carbonisation, avec du bois d'eucalyptus issu des plantations énergétiques situées aux alentours du village d'Ankitsaka dans la région Diana. Elle est basée sur des expérimentations réalisées sur le terrain. L'humidité moyenne du bois était de $36 \%$ lors de la carbonisation. Deux versions du four GMDR ont été testées.

Les résultats obtenus établissent que les rendements massiques de carbonisation sont de l'ordre de $34 \%$, pour les deux versions du four, ce qui place la GMDR parmi les techniques de carbonisation les plus efficaces. Ils montrent également, pour la version la moins coûteuse (environ $3000 €)$, la possibilité d'éliminer une grande partie du méthane produit par le procédé, dès sa sortie de la chambre de carbonisation du four. Ce faisant, les émissions sont de l'ordre de 4,3 kg de méthane par tonne de charbon de bois produite, ce qui place la GMDR parmi les techniques artisanales de carbonisation aux émissions de méthane les plus faibles. L'utilité environnementale de cette technique de carbonisation est confirmée en estimant les émissions de GES évitées ; en remplacement d'une technique de carbonisation à très bas rendement (12\%), la GMDR évite l'émission de 13 Teq $\mathrm{CO}_{2}$ par tonne de charbon de bois produite.

Ce four à la technicité faible offre également une longue durée de vie, diminue le temps durant lequel la présence des charbonniers est requise pour la gestion de la carbonisation et permet l'obtention de rendements élevés et stables, même avec une charge à l'humidité élevée.

$\mathrm{Au}$ vu des avantages techniques et environnementaux décrits ci-dessus, il s'avère que la GMDR recèle un potentiel d'utilisation important, à Madagascar et en Afrique subsaharienne. Afin de le favoriser, des développements sont en cours pour réduire encore le coût de construction du four. Ces avantages ne doivent cependant pas masquer la nécessité de l'associer à une structure de gestion performante et à un volet de formation approprié et suffisant.

\section{Remerciements}

Les résultats présentés dans cette étude ont été obtenus dans le cadre du Programme d'appui à la gestion de l'environnement (PAGE) de la coopération allemande (GIZ) à Madagascar.

\section{Références bibliographiques}

AMS-III.BG, Version 3.0, 2013. Emission reduction through sustainable charcoal production and consumption. Clean Development Mechanism (CDM) methodology booklet, UNFCCC. https://cdm.unfccc.int/methodologies/DB/ MVOAXD3LGD4ZJEKEERCT39ZLJ3JZA0

Burny P., Montagne P., Crehay R., Randrianja H., Rasamindisa A.-M., 2008. La production de charbon de bois à Madagascar: amélioration technique et économique. In : Petrescu M., Ruxandra M. (éds). Environmental policies and legislation. Gembloux, Belgique, Presses agronomiques de Gembloux, 31-40. https://orbi.uliege.be/handle/2268/113466 Doat J., Pétroff G., 1975. La carbonisation des bois tropicaux. Bois et Forêts des Tropiques, 159 : 55-72. http:// revues.cirad.fr/index.php/BFT/article/view/19272

FAO, 2017. The charcoal transition: greening the charcoal value chain to mitigate climate change and improve local livelihoods. Rome, Italy, FAO, 178 p. http://www.fao.org/3/ a-i6935e.pdf

Girard P., 1992. Techniques et matériels de carbonisation. Contrôle de performances. Bois et Forêts des Tropiques, 232 : 53-65. http://revues.cirad.fr/index.php/BFT/article/ view/19747

Girard P., 2002. Quel futur pour la production et l'utilisation du charbon de bois en Afrique ? Unasylva, 211 (53) : 30-34. http://www.fao.org/docrep/pdf/005/y4450f/y4450f05.pdf

Girard P., Napoli A., 2005. La pyrolyse. In : Schenkel Y., Benabdallah B. (éds). Guide biomasse énergie. $2^{\mathrm{e}}$ édition. Québec, Canada, IEPF, 391 p.

ISO 18122:2015, 2015. Solid biofuels - Determination of ash content. Geneva, Switzerland, International Organization for Standardization.

ISO 18123:2015, 2015. Solid biofuels - Determination of the content of volatile matter. Geneva, Switzerland, International Organization for Standardization.

ISO 18134-2:2017, 2017. Solid biofuels - Determination of moisture content - Oven dry method - Part 2: Total moisture - Simplified method. Geneva, Switzerland, International Organization for Standardization.

Madon G., 2017. Le bois, énergie de première nécessité en Afrique. Une ressource trop souvent négligée. Afrique Contemporaine, 261-262 : 201-222. https://doi. org/10.3917/afco.261.0201

Montagne P., Razafimahatratra S., Rasamindisa A., Crehay R., 2010. Arina, le charbon de bois à Madagascar : entre demande urbaine et gestion durable. Antananarivo, Madagascar, CITE-Cirad, $187 \mathrm{p}$.

Mundhenk P., Gomis O., Sy M. C., 2010. Comparaison des rendements de production de charbon de bois entre la meule traditionnelle et la meule Casamance dans la forêt communautaire de Sambandé. Dakar, Sénégal, PERACOD, 22 p. https://energypedia.info/images/3/38/Meule casamancaise PERACOD Mundhenk.pdf 
Pennise D. M., Slith K. R., Kithinji J. P., Rezende M. E., Raad T. J., Zhang J., et al., 2001. Emission of greenhouse gases and other airborne pollutants from charcoal making in Kenya and Brazil. Journal of Geophysical Research, 106: 24-143-155. https://doi.org/10.1029/2000JD000041

Piketty M. G., Fonseca Morello T., Bouillet J.-P., Laclau J.-P., Behling M., Caldeira Pires A., et al., 2011. Charbon de bois et sidérurgie en Amazonie brésilienne : quelles pistes d'améliorations environnementales ? L'exemple du pôle de Carajas. Paris, France, Agence française de développement, Document de travail 112, 87 p. https://www. afd.fr/fr/charbon-de-bois-et-siderurgie-en-amazonie-bresilienne-quelles-pistes-dameliorations-environnementales-lexemple-du-pole-de-carajas

Riuji Lohri C., Mtoro Rajabu H., Sweeney D. J., Zurbrügg C., 2016. Char fuel production in developing countries - A review of urban biowaste carbonization. Renewable and Sustainable Energy Reviews, 59: 1514-1530. https://doi. org/10.1016/i.rser.2016.01.088

Sanogo C. A., Elhadji Mahamane M. L., Khennas S., Konadji H., Van der plas J. R., Girard P., 2006. Techniques améliorées de carbonisation au Sahel. Ouagadougou, Burkina Faso, PREDAS, $40 \mathrm{p}$.

Schenkel Y., 1999. Modélisation des flux massiques et énergétiques dans la carbonisation du bois en four cornue. Thèse de doctorat, Faculté des sciences agronomiques de Gembloux, Belgique, 328 p.

Schenkel Y., Bertaux P., Vanwijnsberghe S., Carré J., 1997. Une évaluation de la technique de carbonisation en meule. Biotechnologie, Agronomie, Société et Environnement, 1 (2) : 113-124. http://www.pressesagro.be/ojs/index.php/ base/article/view/16

Schenkel Y., Temmerman M., Van Belle J. F., Vankerkove R., 1999. New Indicator for Evaluation of the wood carbonization process. Energy Sources, 21: 935-943. https://doi. org/10.1080/00908319950014308

Shah N., Girard P., Mezerette C., Vergnet A. M., 1992. Woodto-charcoal conversion in a partial-combustion kiln: an experimental study to understand and upgrade the process. Fuel, 71 (8): 955-962. https://doi.org/10.1016/00162361(92)90248-M

Sjølie H. K., 2012. Reducing greenhouse gas emission from households and industry by the use of charcoal from sawmill residues in Tanzania. Journal of Cleaner Production, 27: 109117. https://doi.org/10.1016/j.jclepro.2012.01.008

Sparrevik M., Adam C., Martinsen V., Jubaedah, Cornelissen G., 2015. Emissions of gases and particles from charcoal/ biochar production in rural areas using medium sized traditional and improved "retort" kilns. Biomass \& Bioenergy, 72: 65-73. https://doi.org/10.1016/i.biombioe.2014.11.016
Stocker T. F., Qin D., Plattner G.-K., Tignor M., Allen S. K., Boschung J., Nauels A., Xia Y., Bex V., Midgley P. M. (eds), 2013. Climate Change 2013: The Physical Science Basis. Contribution of Working Group I to the Fifth Assessment Report of the Intergovernmental Panel on Climate Change. Cambridge, United Kingdom, New York, NY, USA, Cambridge University Press, 1535 p. http://www.climatechange2013. org/report/full-report/

Bois et Forêts des Tropiques - Revue scientifique du Cirad

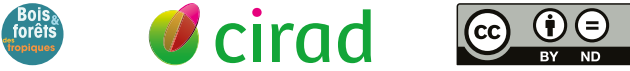

Cirad - Campus international de Baillarguet, 34398 Montpellier Cedex 5, France - Contact: bft@cirad.fr -ISSN: L-0006-579X 